\title{
Fabric Defect Detection Using Adaptive Wavelet
}

\author{
Yang Xue Zhi, Grantham K. H. Pang and Nelson H. C. Yung \\ Department of Electrical and Electronic Engineering \\ The University of Hong Kong \\ E-mail: \{xzyang,gpang,nyung\}@eee.hku.hk
}

\begin{abstract}
This paper studies the adaptive wavelet design for fabric defect detection. In order to achieve translation invariance and more flexible design, the wavelet design focused on nonsubsampled wavelet transform. We design the wavelet filters under the constraints that the analysis filters are power complementary, and the wavelet has only one vanishing moment, which corresponds to a multiscale edge deteter. Based on lattice structure factorization, the design of power complementary filter turn out to be unconstrainted optimization of lattice coefficients. Adaptive wavelets are designed for five kinds of fabric defects in the experiments. Comparing the proposed method with adaptive wavelet design for defect detection based on orthogonal wavelet transform, our design largely improve the ratio of wavelet transform energy between the defect area and the background, and achieve a robust and accurate detection of fabric defects.
\end{abstract}

\section{Introduction}

In textile industry, inspection of fabric defects plays an important role in the quality control. However, the current inspection task is primarily performed by human inspectors and this intensive labour cannot always give consistent evaluation of products. Fabric Automatic Visual Inspection(FAVI) system is an attractive alternative to human vision inspection. Based on advances in computer technology, image processing and pattern recognition, FAVI system can provide reliable, objective and stable performance on fabric defects inspection. For fabric defects detection techniques, there are two main directions. One is statistical texture analysis, which is based on the visual textural properties of the fabric. This approach is weak in small defects detection since the detection primarily depends on statistical texture property. The other direction adopts wavelet transform and addresses fabric defects detection on multiscale analysis. Wavelet analysis, which has the characteristic of preserving the locality of position-frequency event in signal representation[1], can supply a multiscale position-frequency representation of the fabric image. Hence it is more suitable for the analysis and detection of fabric defects. Wavelet transform has been widely applied in fabric defects detection[2-7]. All these work show that defect detection based on wavelet transform has better performance and less computation than statistical texture analysis approaches, especially for some small fabric defects.
There also exist problems in wavelet transform approach As it is expected, after performing wavelet transform on fabric image, defect areas should give significantly larger response than the background in certain scale or some scales to enable easy detection, while the response from the background fabric texture will probabiy disturb the detection of defects and cause false alarm. Jasper et al. [6], and Gong[7] try to solve this problem by adaptive wavelet design for specific fabric texture. They derive an orthogonal wavelet bases directly from the texture data of the defect-free image, such that the wavelet filter gives a close to zero response to that texture, while disturbances in the texture due to noise and defects will produce a nonzero output. Comparing to Daubechies wavelets, the adaptive wavelet bases they designed has achieved better performance in enhancing the response from the defect area. In fact, their method is based on the assumptions that the degree of orthogonality between defect area and background texture is strong and the design freedom of the wavelet filter is large enough to meet the design specification. However, our experiment results has shown that defect area is highly related to background fabric texture. It is not suitable to separate them with orthogonal wavelet transform. Also orthogonal wavelet transform imposes considerable constraints on the wavelet filter design. Furthermore critically sampling of orthogonal wavelet transform destroys shift invariance, which is important for fabric defects detection. In order to obtain a shift invariant decomposition and more freedom on the wavelet bases design, we study the adaptive wavelet design for fabric defect detection which is addressed on nonsubsampled wavelet transform. The experiment results show that, comparing the proposed method with adaptive wavelet design for defect detection based on orthogonal wavelet transform, our design largely improve the ratio of wavelet transform energy between the defect area and the background and achieve a robust and accurate detection of fabric defect.

\section{Nonsubsampled octave band filter bank and discrete wavelet frame}

A nonsubsampled octave band filter banks is shown in Figure 1. $H(z)$ and $G(z)$ denote the z-transform of low-pass filter $h$ and high-pass filter $g$ respectively. The signal is iteratively decomposed on the low-pass analysis channel. Based on this structure, one can devise a discrete time wavelet transform and obtain a redundant signal representation. This overcomplete wavelet representation is also called a discrete wavelet frame. Comparing to critically sampled octave band filter banks, 


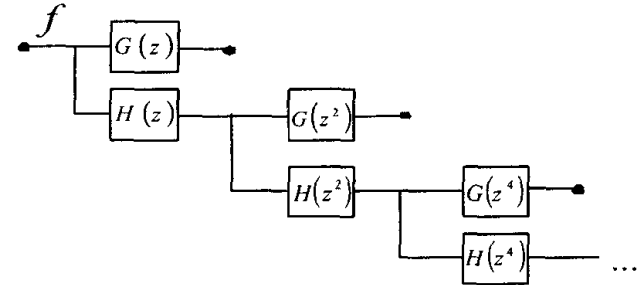

Figure 1. A nonsubsampled octave band filter bank.

nonsubsampled version achieves translation invariance, which is especially desirable in pattern recognition applications, such as fabric defect detection. And nonsubsampled version also has less constraints for perfect reconstruction which allows for more flexible design. This overcomplete signal expansion has been applied in texture classification and segmentation[8,9], multiscale edges detection[10] and singularity detection[11].

The cascade of filtering operations in the nonsubsampled octave band filter bank shown in Figure 1 can be described as the following equivalent filter sequences

$$
\begin{gathered}
H_{i}(z)=H\left(z^{2^{i-1}}\right) H_{i-1}(z)=\prod_{k=0}^{i-1} H\left(z^{2^{k}}\right) \\
G_{i}(z)=G\left(z^{i-1}\right) H_{i-1}(z)=G\left(z^{2^{i-1}}\right) \prod_{k=0}^{i-2} H\left(z^{2^{k}}\right) \\
i=1, \ldots, I
\end{gathered}
$$

Here $H_{0}(z)$ is defined as 1 . In this paper, we use two oriented two-dimension wavelets $\psi_{i}^{1}(x, y)$ and $\psi_{i}{ }^{2}(x, y)$ for fabric defect detection, which are constructed as follows

$$
\begin{gathered}
\psi_{i}^{\prime}\left(z_{1}, z_{2}\right)=G\left(z_{1}^{2^{i-1}}\right) \prod_{k=0}^{i-2} H\left(z_{1}^{2^{k}}\right) H\left(z_{2}^{2^{k}}\right) \\
\psi_{i}{ }^{2}\left(z_{1}, z_{2}\right)=G\left(z_{2}^{2^{i-1}}\right) \prod_{k=0}^{i-2} H\left(z_{1}^{2^{k}}\right) H\left(z_{2}^{2^{k}}\right) \\
i=1, \ldots, I
\end{gathered}
$$

where $\psi_{i}{ }^{\prime}\left(z_{1}, z_{2}\right)$ and $\psi_{i}{ }^{2}\left(z_{1}, z_{2}\right)$ have horizontal and vertical orientation respectively, and $i$ is the scale index. If the highpass filter $G(z)$ has exact one zero at $z=1$, then we can detect multiscale edges in an image based on wavelet transform modulus[10].

\section{Wavelet filter design}

Based on nonsubsampled octave band filter banks, we study the filter design under the constraint that $H(z)$ and $G(z)$ are power complementary, i.e., $H(z)$ and $G(z)$ satisfy

$$
H(z) H\left(z^{-1}\right)+G(z) G\left(z^{-1}\right)=1
$$

which corresponds to tight frames in the space of square summable sequences[13].
We also impose the following constraints on $H(z)$ and $G(z)$

$$
H(-1)=0 \quad \text { and } \quad G(1)=0
$$

where $H(-1)=0$ is required for meeting regularity constraint. By choosing $G(1)=1$, we will construct a wavelet with one vanishing moment, which is equivalent to a mutliscale edge detecter.

We have tried two approaches in designing filters $H(z)$ and $G(z)$ satisfying constraints (3) and (4). The first is based on spectral factorization, and the second is based on lattice structures. Spectral factorization approach[12] designs the autocorrelation function $H(z) H\left(z^{-1}\right)$ of the low-pass filter $H(z)$, then perform spectral factorization on $H(z) H\left(z^{-1}\right)$ and $G(z) G\left(z^{-1}\right)$ to obtain filters $H(z)$ and $G(z)$. For meeting the design specification, optimization procedure will be performed on the coefficients of $H(z) H\left(z^{-1}\right)$. However, spectral factorization approach becomes increasingly inaccurate and time consuming when the order of the filter autocorrelation function is high. Moreover, optimization procedure based on coefficients of $H(z) H\left(z^{-1}\right)$ is a constrainted optimization since those coefficients which lead to complex filters should be avoided.

An alternative and numerically well-conditioned design procedure is based on lattice structure[14], which perform a cascade-form factorization for $[H(z) G(z)]$

$$
\left[\begin{array}{c}
H(z) \\
G(z)
\end{array}\right]=R_{m} \Lambda(z) R_{m-1} \Lambda(z) \ldots R_{1} \Lambda(z)\left[\begin{array}{c}
\cos \theta_{0} \\
\sin \theta_{0}
\end{array}\right]
$$

where

$$
R_{k}=\left[\begin{array}{cc}
\cos \theta_{k} & \sin \theta_{k} \\
-\sin \theta_{k} & \cos \theta_{k}
\end{array}\right] \text { and } \Lambda(z)=\left[\begin{array}{ll}
1 & 0 \\
0 & z^{-1}
\end{array}\right]
$$

With this design method, power complementary property (3) is structurally satisfied. And under the constraints (4) in this factorization structure, we will have $m-1$ independent free parameters to choose in designing filters with length $m+1$. With lattice structure factorization, the design of power complementary filters turns out to be unconstrainted optimization of the lattice coefficients.

\section{Objective function and optimization method}

The goal of adaptive wavelet design is to maximize the ratio of wavelet transiorm energy between defect area and the defectfree background at certain scale, such that the defect area can be easily segmented out of the background by a simple threshold classifier. Based on this assumption, our objective function is designed as follows

$$
J=\frac{\mu_{D}}{\mu_{N}}
$$

where $\mu_{D}$ is the mean energy of wavelet transform coefficients at certain scale within defect area and $\mu_{N}$ is the mean energy of 
wavelet transform coefficients of the defect-free image, at same scale as $\mu_{D}$

Based on lattice structure factorization, the maximization of the objective function $J$ with respect to lattice rotation angles is complicated since $J$ is not strictly convex or concave, having a number of local maxima. Several optimization techniques have been tried, they are quasi-Newton method, ring algorithm[15] and Nelder-Mead Simplex algorithm. Quasi-Newton method is selected because its convergence is relatively fast, especially for high order lattice structure.

\section{Experiment results}

Adaptive wavelet filters have been designed for the detection of five kinds of fabric defects. These defects are Wrong Draw, Broken End, Mispick, Thin Bar and Slack End. All the design are performed on scale 2 . Filter design in scale 1 does not lead to satisfactory result, since the wavelet we design is equivalent to multiscale edge detecter, scale 1 is too fine and noisy. Designing on scale larger that 2 also can yield large ratio of wavelet transform energy between defects area and the background, while the location of the defect area is not as precise as in scale 2, since the resolution become lower and filter length become longer. In choosing the filter length, we prefer shorter filter as it can provide a more accurate location of the defect area and lower computational cost, but it also provides less free parameters for design. In designing the filter, we evaluated a number of filter sizes which range from 3 to 20 and selected the best candidate. Normally filter length larger that 12 can lead to a good optimization result. In our experiment, it is found that, optimization based on the wavelet transform component, whose direction is along with the orientation of the defect distribution (nearly horizontal or vertical direction), can result in a larger ratio of wavelet transform energy between defects area and the background and a more precise location of the defect area than on the other component. In fact, there are two kinds of edges in locating the defect area. One is the edges between the defect area and the background fabric image, and the other kind of edges is the texture edges of texture primitives within the defect area, which is different from the edges of background fabric texture in size and orientation. Defect areas normally have small width. If the defect area is not strongly different from the background in gray scale, it is very difficult to locate the edges between the defect area and the background. Alternatively, along the orientation of the defect area, we have enough size to evaluate the texture properties of defect area in that direction. Edges within defect area and the background response differently to edge detective wavelet, and this difference can be enlarged by wavelet design, which leads to a robust detection of the defect area.

Using adaptive wavelet filter, the detection results for fabric defects Mispick and Broken End are shown in Figure 2 and Figure 3. For the purpose of displaying the energy of the wavelet transform, their values have been linearly mapped to the gray level of interval $0 \sim 255$. The threshold is selected as $\mu+3.5 \sigma$, where $\mu$ and $\sigma$ are the mean and standard deviation of the energy of same wavelet transform on defect-free image. As the experiment results show, the adaptive wavelet response much differently to the defect area and the defect-free background area, and we can achieve good detection of the defect areas.
For comparative study, we also implement the orthogonal wavelet design for fabric defect detection. The design procedure here follows along the same lines as Jasper[6] and Gong[7]. Lattice structure is used for orthogonal wavelet design[7]. Experiment results show that, comparing with Daubechies wavelet, the response from defect area in detail space is enhanced, while the disturbance from background is still not be efficiently suppressed, and it is difficult to find a suitable threshold to segment the defect areas out of the background. This result imply that the defect area is closely related to the background fabric texture, and it is not feasible to separate them by orthogonal wavelet transform.

It has been verified in our experiment that equation (6) is closely proportional to the detection performance, that is, larger value corresponds to more accurate and easier detection of fabric defects. Comparison based on this criterion for adaptive wavelet design of nonsubsampled wavelet transform, adaptive wavelet design of orthogonal wavelet transform and Daubechies wavelet is shown in Table 1. The filter length of the quadrature mirror filter for adaptive wavelet in orthogonal wavelet transform is 12 , and we choose the detail space in which the value of equation (6) is larger than the other two detail spaces.

\section{Conclusions}

In this work, we study the wavelet design for fabric defect detection based on nonsubsampled wavelet transform. Comparing with orthogonal wavelet transform, nonsubsampled wavelet transform has the advantages of translation invariance and more flexible for the wavelet design. The adaptive wavelets we design largely improve the ratio of wavelet transform energy between defect areas and the background fabric texture and achieve robust and accurate detection of fabric defects. From our experiment results, we can conclude that it is not suitable to segment the defect area out of the background by orthogonal wavelet transform since they are highly related, and the adaptive design of orthogonal wavelet for defect detection has limited performance. For power complementary filter design, lattice structure factorization formulate the design as unconstrainted optimization of the lattice coefficients, which is more efficient than spectral factorization method. We also find that, by using a wavelet with the ability of multiscale edge detection, it is an efficient approach to locate the defect areas based on the wavelet transform response on the texture edges along the defect orientation within he defect area and the background fabric texture.

\section{References}

[1] S. Mallat, "A theory for multiresolution signal decomposition: the wavelet representation", IEEE Transactions On PAMI, Vol. 11, no. 7, July 1989, pp. 674-693

[2] H. Sari-Sarraf, J.S. Goddard, "Vision system for on-loom fabric inspection", IEEE Transactions on Industrial Applications, Vol.35 6,1999, pp.1252-1259.

[3] G. J. Vachtsevanos, M. Mufti, J.L. Dorrity, "Method and apparatus for analyzing an image to detect and identify defects", United States Patent, Patent No. 5815198, Sep. 29,1998 . 
[4] S. Kim, M. H. Lee, K. B. Woo, "Wavelet analysis to fabric defects detection in weaving processes", Proceedings of the IEEE International Symposium on Industrial Electronics, Vol.3,1999, pp.1406-1409.

[5] G. Lambert, F.Bock, "Wavelet methods for texture defect detection", Proceedings of the IEEE International Conference on Image Processing, Vol.3,1997, pp.201204.

[6] W. J. Jasper, S. J. Garnier, and H. Potlapalli, "Texture characterization and defect detection using adaptive wavelets", Optical Engineering, 35(11),1996, pp.31833149.

[7] YuNan Gong, "Study on Image Analysis of Fabric Defects", Ph.D. dissertation, China Textile University, 1999.

[8] M. Unser, "Texture Classification and Segmentation Using Wavelet Frames", IEEE Transactions on Image Processing, Vol.4 No. 11, Nov. 1995, pp.1549-1560.

[9] A. Laine and J. Fan, "Frame Representations for Texture Segmentation", IEEE Transactions on Image Processing, Vol.5 No. 5, MAY. 1996, pp.771-780.

[10] S. Mallat and S. Zhong, "Characterization of Signals from Multiscale Edges", IEEE Transactions on PAMI, Vol. 14 No. 7 , July. 1992, pp.710-732
[11] S. Mallat and W. L. Hwang, "Singularity Detection and Processing with Wavelets", IEEE Transactions on Information Theory, Vol. 38 No. 2, March. 1992, pp.617643.

[12] Z. Cvetkovic and M. Vetterli, "Discrete-Time Wavelet Extrema Representation: Design and Consistent Recognition", IEEE Transactions on Signal Processing, Vol. 43 No. 3, pp.681-693, March. 1995, pp.681-693.

[13] Z. Cvetkovic and M. Vetterli, "Oversampled Filter Banks", IEEE Transactions on Signal Processing, Vol. 46 No. 5, March. 1998, pp.1245-1255.

[14] P.P. Vaidyanathan and Z. Doganata, "The Role of Lossless Systems in Modern Digital Signal Processing: A Tutorial", IEEE Transactions on Education, Vol. 32 No. 3, Aug. 1989, pp.181-197.

[15] P. Desarte, B. Macq and D. T. M. Slock, "Signal-Adapted Multiresolution Transform for Image Coding", IEEE Transactions on Information Theory, Vol. 38 No. 2, March. 1992, pp.897-904.

[16] P.P. Vaidyanathan and P. Q. Hoang, "Lattice Structures for Optimal Design and Robust Implementation of TwoChannel Perfect-Reconstruction QMF Banks", IEEE Transactions on ASSP, Vol. 36 No. 1, Jan. 1988, pp.8194

Table 1. Comparison based on equation (6) for adaptive wavelet design of nonsubsampled wavelet transform, adaptive wavelet design of orthogonal wavelet transform and Daubechies wavelet(D12).

\begin{tabular}{|c|c|c|c|}
\hline Fabric Defect & $\begin{array}{l}\text { Adaptive wavelet } \\
\text { design for } \\
\text { nonsubsampled WT }\end{array}$ & $\begin{array}{l}\text { Adaptive wavelet } \\
\text { design for } \\
\text { orthogonal WT }\end{array}$ & $\begin{array}{c}\text { Daubechies wavelet } \\
\text { (D 12) }\end{array}$ \\
\hline Wrong Draw & 2.81 & 1.57 & 1.04 \\
\hline Broken End & 4.39 & 2.24 & 1.39 \\
\hline Slack End & 3.19 & 1.67 & 1.11 \\
\hline Thin Bar & 3.65 & 2.18 & 2.15 \\
\hline Mispick & 11.66 & 1.70 & 1.62 \\
\hline
\end{tabular}

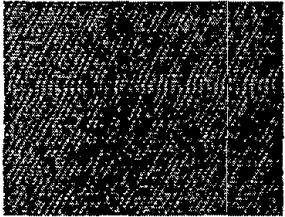

(a)

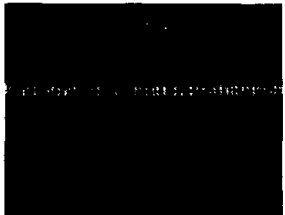

(b)

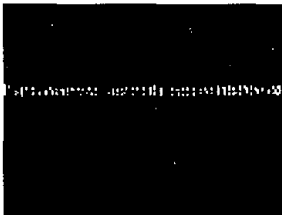

(c)

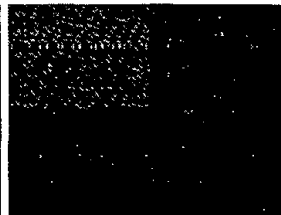

(d)

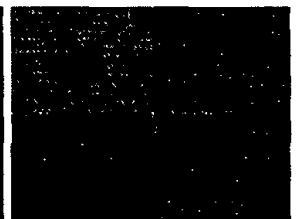

(e)

Figure 2. (a) Original fabric image, containing defect Mispick (b) Nonsubsampled wavelet transform in horizontal direction at scale 2, using adaptive wavelet (c) Thresholding of (b). (d) Orthogonal wavelet transform, using adaptive wavelet. (e) Orthogonal wavelet transform, using Daubechies wavelet(D12).

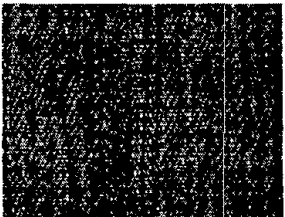

(a)

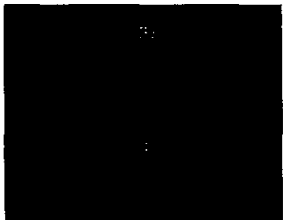

(b)

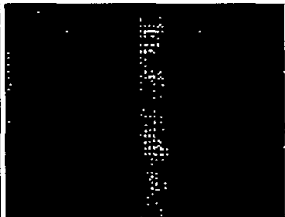

(c)

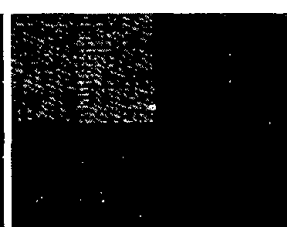

(d)

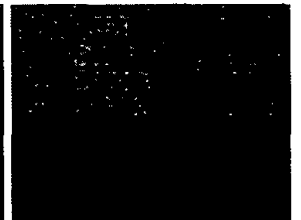

(e)

Figure 3. (a) Original fabric image, containing defect Broken End. (b) Nonsubsampled wavelet transform in vertical direction at scale 2, using adaptive wavelet (c) Thresholding of (b). (d) Orthogonal wavelet transform, using adaptive wavelet. (e) Orthogonal wavelet transform, using Daubechies wavelet(D12). 